\title{
MIE SCATTERING AND THE MARTIAN ATMOSPHERE
}

\author{
WALTER G. EGAN and KENNETH M. FOREMAN \\ Research Dept., Grumman Aerospace Corporation, Bethpage, N.Y., U.S.A.
}

\begin{abstract}
It has been suggested that the discrepancy between radio occultation determinations of the Martian atmospheric surface pressure (3.8 to $7 \mathrm{mb}$ ) and those deduced from optical polarization measurements and a simple Rayleigh atmosphere model (about $10 \mathrm{mb}$ ) are the results of submicron sized aerosols in the Martian atmosphere. Based on observed viewing angle dependence of the polarization of the Martian disk in the visual range, a Mie scattering analysis has been made utilizing the measured complex index of refraction of limonite and bulk solid $\mathrm{CO}_{2}$. The results of this study indicate that limonite aerosols alone are unsatisfactory to explain the viewing angle observations, whereas solid $\mathrm{CO}_{2}$ (and $\mathrm{H}_{2} \mathrm{O}$ ice) aerosol spheres, having a dominant particle radius range between 0.28 and $0.35 \mu$, could bring planetary and laboratory observations into compatibility. It is suggested, further, that solid $\mathrm{CO}_{2}$ aerosols could explain limb brightening in the blue spectral range. Various distributions of solid $\mathrm{CO}_{2}$ and $\mathrm{H}_{2} \mathrm{O}$ Mie particles with radii up to $0.35 \mu$ show an opposition effect. However, the role of these aerosols in explaining the Mars opposition observations is very dependent on the optical properties of the underlying Mars surface material.
\end{abstract}

Measurements of the Martian atmospheric surface pressure by the radio frequency occultation determinations of Mariners IV, VI, and VII yield values between 3.8 and $7 \mathrm{mb}$ (Kliore et al., 1966; Kliore, 1969). The spectroscopic data have been in reasonably good agreement, yielding a value of the order of $10 \mathrm{mb}$ (Kaplan et al., 1964). Still another technique, polarization measurement, has been yielding pressure values that are too large in comparison to the previously noted methods (Dollfus and Focas,1966). Part of the problem with polarization lies in the nature of the laboratory specimen used for surface simulation as well as in the planetary model used for interpretation of laboratory polarization data compared to earth-based observations of the planet. Polarization is an extremely sensitive indicator of atmospheric as well as surface optical effects (Dollfus and Focas, 1966).

It cannot be emphasized too strongly that average gross features are involved in both telescopic observations and surface simulations, and as large a scale as possible of laboratory surface simulation is necessary (Egan, 1967). Further, simultaneous consideration of many aspects of surface simulation, e.g., photometry and polarimetry and thermal effects, etc., is required (Egan, 1969).

The polarimetric results of Dollfus have been examined and refined analysis yields an average surface pressure of about $20 \mathrm{mb}$ (O'Leary and Pollack, 1969), a factor of 3 or more greater than the 4 to $7 \mathrm{mb}$ value range deduced from Mariner spacecraft data. Our analyses based on further refinements of our large scale surface simulation experimental data have indicated a total surface pressure of about $10 \mathrm{mb}$ for a simple Rayleigh atmosphere model (Foreman, 1969). Thus, an aerosol contribution equivalent to the optical properties of the order of $5 \mathrm{mb}$ of atmosphere appears indicated. Various investigators (e.g., Kuiper (1964), Rea and O'Leary (1965), Coulson (1969)) have calculated the Mie scattering effects of terrestrial, water ice, and silica aerosols. 
O'Leary (1967) has questioned the significance of any surface simulation attempt in view of the numerous possibilities for aerosol effects. Based on Mars observations and various optical models, our opinion is that the best approach still lies in a laboratory simulation as close as possible to Mars data and with an allowance being made for a generally small atmospheric perturbing effect.

More extensive Mie scattering calculations for estimating the Mars aerosol effect have become possible this past year with the determination of the complex index of refraction of Venango County (Pennsylvania) limonite (Egan and Becker, 1969) and of bulk solid $\mathrm{CO}_{2}$ (Egan and Spagnolo, 1969) in the spectral range between 0.35 and $1.0 \mu$. Both substances are potential candidate constituents of Mars aerosols. It is found that the refractive index of solid $\mathrm{CO}_{2}$ is close to that of $\mathrm{H}_{2} \mathrm{O}$ ice. Therefore, computations for dry ice may be considered as also approximately valid for $\mathrm{H}_{2} \mathrm{O}$ ice particles.

This report summarizes a particular aspect of polarization that appears to permit an inference of the nature of aerosols in the Martian atmosphere; a Mie scattering computer program designed and run by Mrs. Jaylee Mead had supplied us with the single scatterer data with which this study has been concerned.

Polarization observations by Dollfus of the central light 'desert' regions of Mars, in the visual spectral range, exhibit a small but real variation of polarization with viewing angle (Figure 1) (Dollfus, 1966). This is of the order of $0.1 \%$ to $0.2 \%$ based

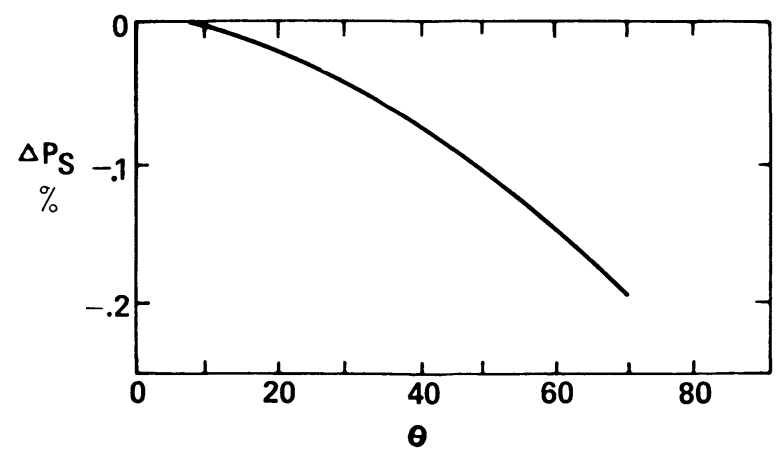

Fig. 1. Mars oblique polarization difference (Dollfus, 1965) $\Delta P_{S}=P_{\theta}{ }^{\circ}-P_{0} \circ$.

on the most updated information analyzed by Dollfus (Cann et al., 1965). In the course of our simulation experiments, we also have discerned a comparable real variation of polarization with viewing angle (and wavelength dependent), however, of opposite sign from Dollfus' observations (Egan and Foreman, 1968). A possible explanation for this subtle paradox is the existence of Mie scatterers in the Martian atmosphere which could produce sufficient negative polarization to bring the particulate limonite surface simulation data into agreement with the planetary characteristics. Rayleigh 
scattering of atmospheric molecules will only produce positive polarization, and, thus, cannot be expected to explain the problem. However, any atmospheric Rayleigh scattering polarization would have to be compensated for additionally by Mie scattering.

The change of Martian polarization with viewing angle shown in Figure 1 amounts to a few tenths of a percent difference in polarization between the center of the disk and the limb, for a particular phase angle, with the limb having the lower polarization.

Our laboratory simulation for the limonite particulate specimen that most closely matches the polarization-brightness characteristics of the bright regions of Mars (i.e., Venango County, Pennsylvania limonite (Egan, 1969)) indicates an oblique polarization difference between 60 degrees and 0 degrees viewing angles for three wavelengths as shown by Figure 2. The data points for a range of phase angles between 5 degrees

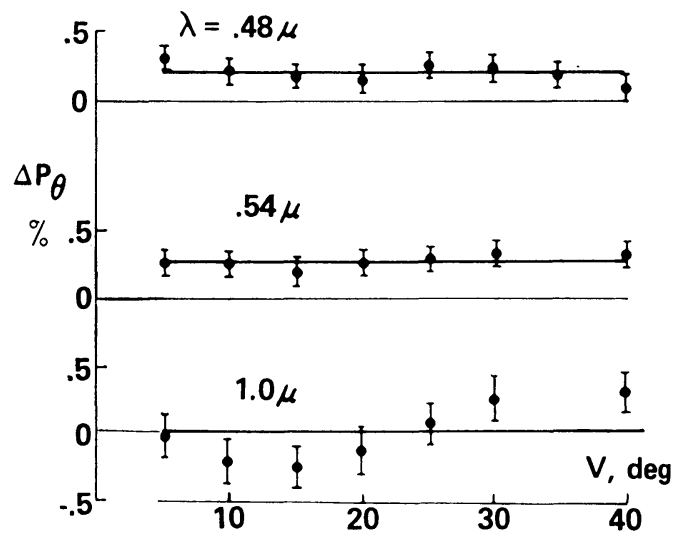

Fig. 2. Oblique polarization difference Venango limonite $\Delta P_{\theta}=P_{60}=-P_{0}$.

and 40 degrees fall on either side of the rms average values shown. The data accuracy is higher for the $0.48 \mu$ and $0.54 \mu$ wavelengths because the photomultiplier sensor signal to noise ratio is greater than for the one used at $1.0 \mu$. The approximate spectral variation of rms average values are shown in Figure 3. In the visual range $(0.54 \mu)$, the limonite specimen will be in agreement with the Martian data if the aerosol component produces a decreasing polarization (i.e., negative $\Delta P$ ) with increasing viewing angle, at any particular phase angle. (This trend also should hold true for all phase angles.)

Mie scattering by individual particles is dependent on the phase angle, $V$, not viewing angle, $\theta$, except when the scatterers exist in a layer above a planet's surface. In this latter situation, as the viewing angle increases, the atmospheric and aerosol layer optical path length increases; the polarization contribution of a widespread aerosol layer can become relatively large with viewing toward the limb. 


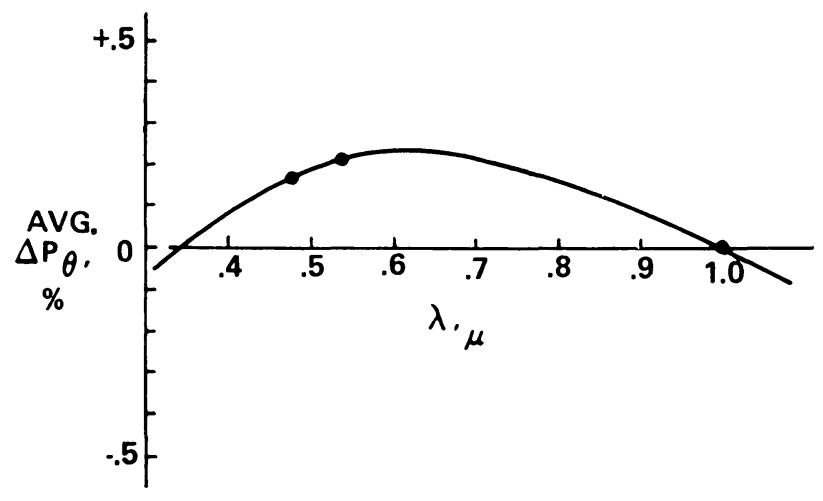

Fig. 3. Average oblique polarization difference - Venango limonite.

The results of the Mie scattering calculations* (simple spheres and single scattering) for Venango County limonite and bulk solid $\mathrm{CO}_{2}$ at $0.54 \mu$ and $1.0 \mu$ are summarized in Figure 4. In this illustration is shown the variation of the $\sum_{r_{0}}^{r} B_{r} P_{r}$ product term with largest particle radius, $r$, for a flat (i.e., equal number) distribution of particle sizes between $r_{0}$ and $r\left(r_{0}\right.$ is $0.005 \mu$ radius and $r$ can be examined up to a maximum of $0.5 \mu$ ). $\left(\sum_{r_{0}}^{r} B_{r} P_{r}\right.$ represents the relative polarization of Mie aerosols, and is the numerically integrated product of relative brightness and polarization for integrating increments of particle radii, $\Delta r$, of $0.005 \mu$. The summation is for all particle sizes up to the maximum, $r$, considered.) This product term is proportional to the polarization contribution of Mie aerosols to the total polarization characteristic of the planet, which also includes the atmosphere and the surface contributions. The curves are shown for a phase angle of 40 degrees.

Additional results were computed for various non-flat distributions (e.g., Gaussian, step rise-exponential decay) and were found to produce virtually no variation in qualitative results although some detailed quantitative differences were evident.

It is apparent that the Venango County limonite produces a negative polarization term at $0.54 \mu$ and zero polarization at $1.0 \mu$ (as required by Figure 3 ) almost uniquely at a dominant particle radius of about $0.27 \mu$. However, additional data, not presented, but generated by our computer study, shows that at other phase angles between 10 degrees and 30 degrees, this dominant particle size yields positive polarization at $\lambda=0.54 \mu$. Therefore, we can conclude that limonite is not a good choice, in itself, for Martian aerosols, at least under Mie scattering theory constraints.

For solid $\mathrm{CO}_{2}$, the situation appears different. In the particle size range between about 0.28 and $0.35 \mu$ radius, the viewing angle variation of polarization difference, $\Delta P$, is of the correct sign and magnitude at the two wavelengths. (It should be noted

\footnotetext{
* Provided by Mrs. Jaylee Mead in private communications, July-October, 1969.

12-P.A.
} 


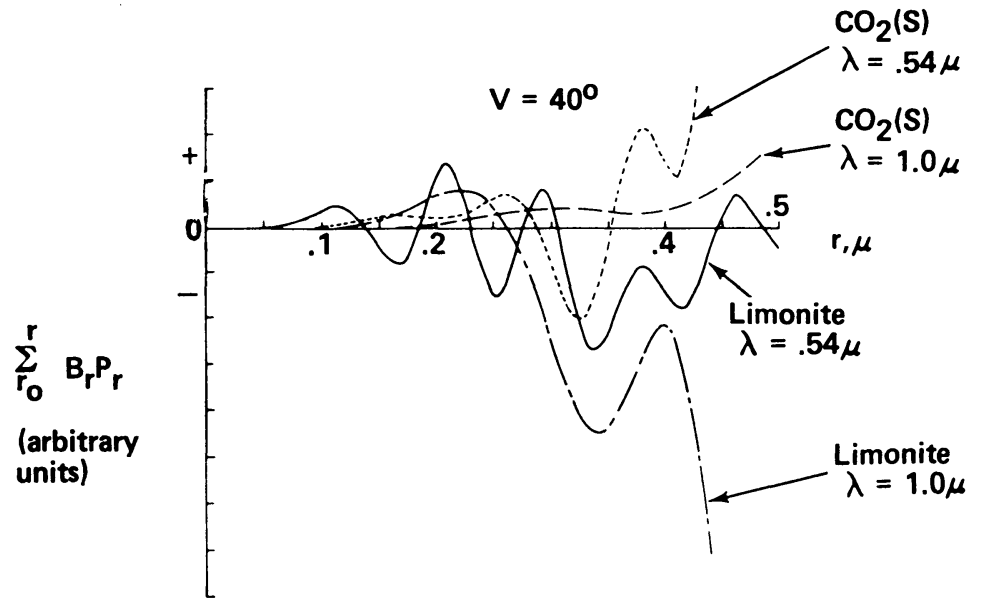

Fig. 4. Integrated product of brightness and polarization vs. Mie scatterer size.

that for $\lambda=1.0 \mu$, we must subtract the summation ordinate value for all particle radii sizes smaller than $0.28 \mu$ from the value at larger sizes and this results in a virtually zero polarization term for dominant particle radii between $0.28 \mu$ and $0.35 \mu$, as required.)

If we examine the photometric properties of solid $\mathrm{CO}_{2}$ and limonite as a function of Mie particle radii at a 0 degrees phase angle, for instance, Figure 5 , it is evident that the solid $\mathrm{CO}_{2}$ particles are generally an order of magnitude brighter in the blue $(0.365 \mu)$ than in the red $(0.740 \mu)$, and increases in brightness with particle size (up to at least $r=0.5 \mu$ ). Limonite aerosols, however, generally do not exhibit a strong color differentiation, and, in many size ranges are of the same order of brightness. Thus, if solid $\mathrm{CO}_{2}$ aerosols of submicron size exist at the limbs of Mars, they could be expected to contribute to the commonly observed limb brightening in the blue portion of the spectrum.

In Figure 6, a further examination of solid $\mathrm{CO}_{2}$ aerosols for other phase angles in the visible region shows that the particle size range between $0.28 \mu$ to $0.35 \mu$ remains compatible in sign, although varying, to some degree, in magnitude for the smaller phase angles.

Continuing to explore the photometric properties of solid $\mathrm{CO}_{2}$ aerosols, in association with a Venango County limonite simulated surface, as a function of wavelength, one can see from Figure 7: (1) a 1-2 mm particulate limonite surface, at a wavelength of $0.54 \mu^{*}$ has an opposition effect close to that observed by O'Leary (1967) for Mars but above that inferred from de Vaucouleurs (1964); (2) solid $\mathrm{CO}_{2}$ aerosols spheres, with a flat size distribution between $0.005 \mu$ radius and approximately $0.33 \mu$, show an opposition effect particularly at the shorter wavelengths. It should be noted that in

\footnotetext{
* Currently, data available are for this wavelength only.
} 


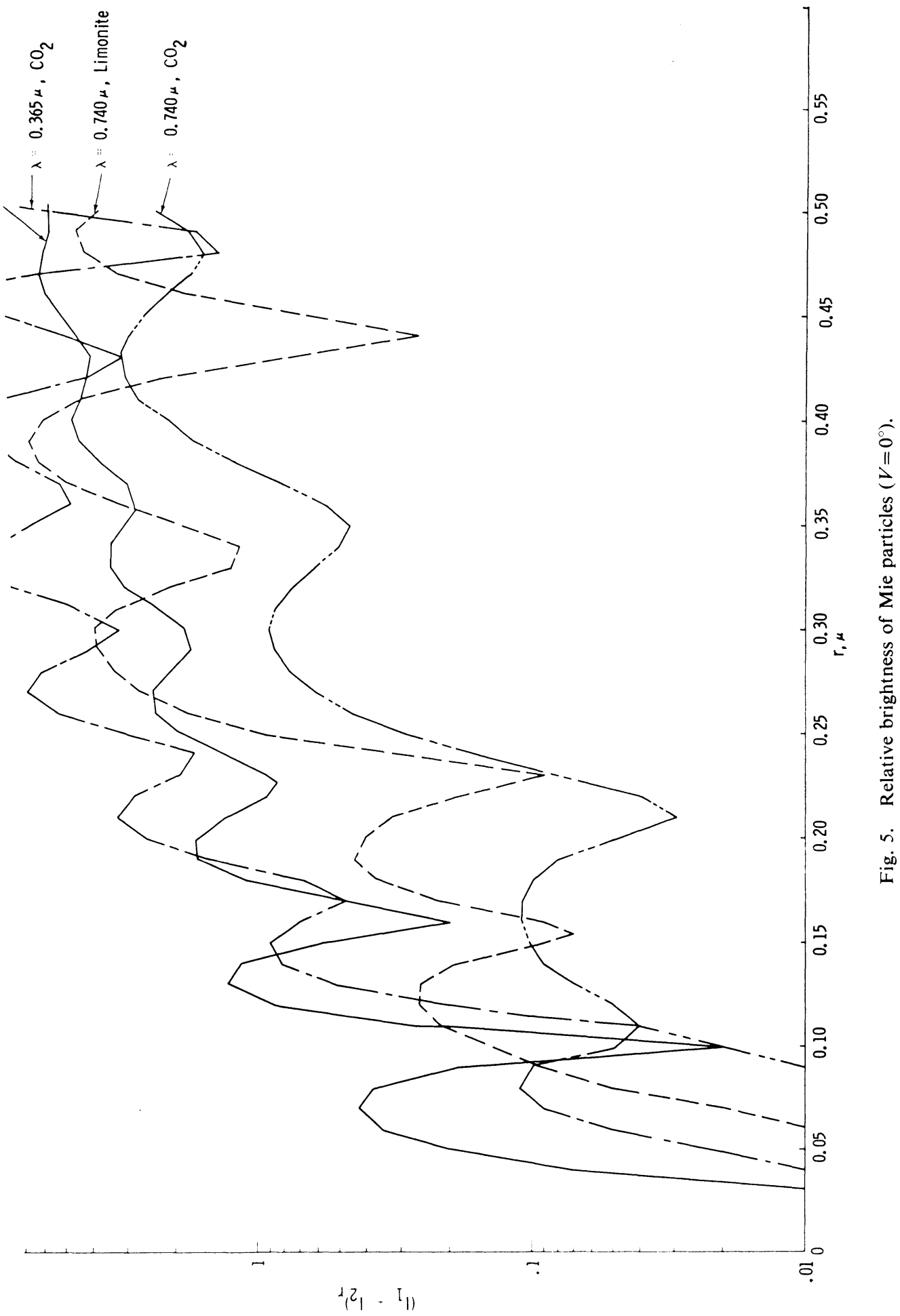




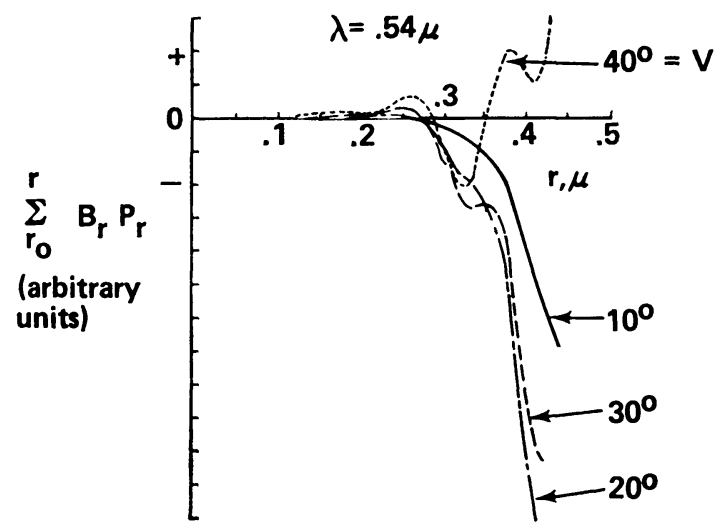

Fig. 6. Integrated product of brightness and polarization vs solid $\mathrm{CO}_{2}$ particle size and phase angle.

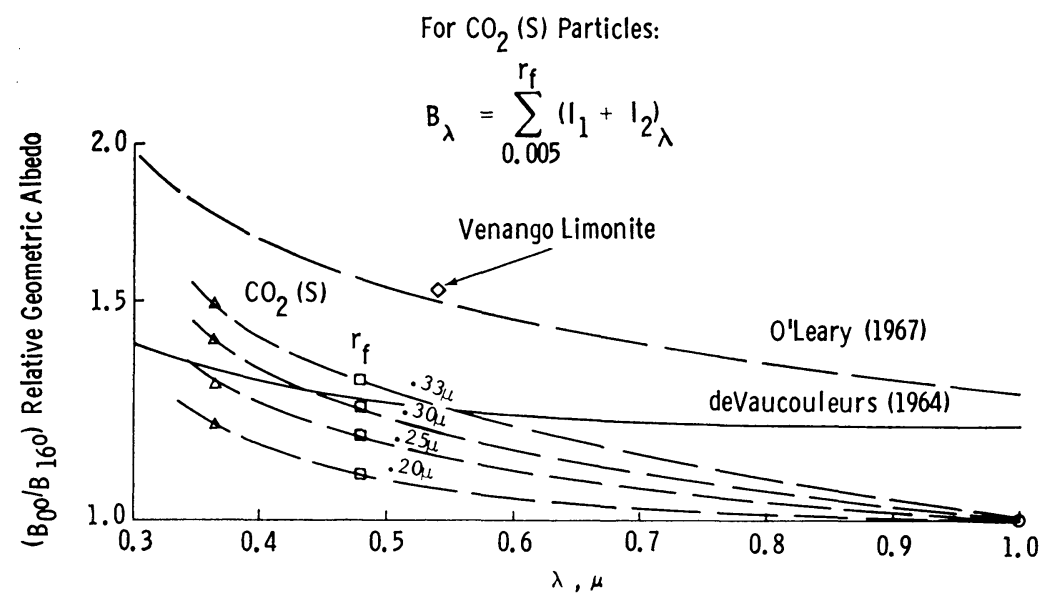

Fig. 7. Opposition effect: Mars observations compared to 1-2 $\mathrm{mm}$ limonite specimens and Mie calculations for solid $\mathrm{CO}_{2}$.

the solid $\mathrm{CO}_{2}$ particle size range inferred by our previous polarization considerations (i.e., $\sim 0.300$ to $0.325 \mu$ radius), the opposition effect may be as large as 1.80 for $\lambda=0.365 \mu$. In numerical computations of Mie scattering photometric characteristics, it is important to use small radii increments $(\Delta r \approx 0.005 \mu)$ for the particles so that the fine details in the results will not be obscured. In addition, it is advisable to examine phase angles greater than the 16 degrees reference angle, usually employed for opposition analyses, for possible secondary maxima in the opposition effect curve; we have found no such violation of the opposition effect in our analyses of computer results for the $\sim 0,30 \mu$ particle radius size deduced for solid $\mathrm{CO}_{2}$. 
It should be noted that if other wavelength determinations with limonite surface specimens similarly follow the trend (Figure 7) of the Mars opposition observations, then the need for aerosol particles to explain the opposition phenomena are considerably lessened. Additional studies of opposition effect are discussed in the other conference paper by Mead (1970). Her results are based on a particular and different set of assumptions, but her conclusions do not conflict with those of this paper which are based on a limonite surface simulation model that closely matches the Martian observations.

Figure 8 presents the variation of vapor pressure of solid $\mathrm{CO}_{2}$ with temperature, and an indication of the equilibrium temperatures between the solid and a pure gaseous $\mathrm{CO}_{2}$ atmosphere. For an atmospheric surface pressure of $7 \mathrm{mb}$, near the

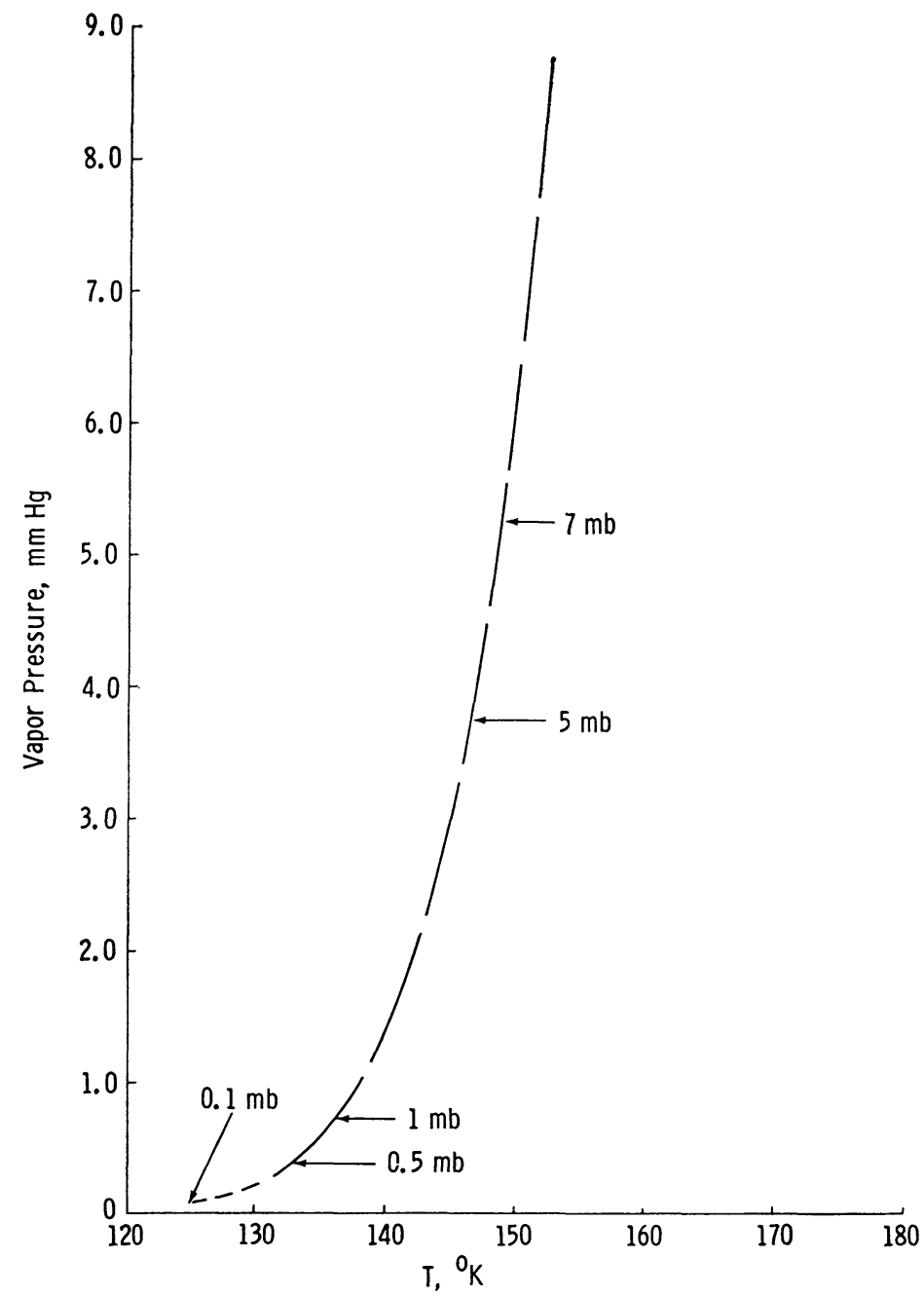

Fig. 8. Vapor pressure of solid $\mathrm{CO}_{2}$ (NBS Circular No. 564, revised). 
Kliore et al. inferred surface pressure (Kliore et al., 1966; Kliore, 1969), the equilibrium temperature is almost $149 \mathrm{~K}$. Radiation-dominated models of the Martian atmospheric dynamics have been studied by Gierasch and Goody (1967/1968) and earth type convective models by Leovy and Mintz (1966).

Profile models of the Martian atmosphere have temperature distributions which depend upon the assumed condition of surface temperature (Gierasch and Goody, 1967/1968; Leovy and Mintz, 1966; Johnson, 1965). Except for polar and high winter latitudes, the near-surface temperature generally does not seem to approach a low enough value to result in dry ice production. However at altitudes where the atmospheric pressure is of the order of a magnitude lower or less (i.e., at $10 \mathrm{~km}$ or above) atmospheric temperatures may become low enough because of atmospheric radiative and dynamic processes to coincide with the temperature at which solid $\mathrm{CO}_{2}$ will form, as indicated by the phase curve. The 1969 Mariner spacecraft photographs (Leighton et al., 1969) show that clouds do occur at altitudes of approximately 15 to $40 \mathrm{~km}$ above the Martian surface and it is reasonable to consider these clouds as consisting of solid $\mathrm{CO}_{2}$ as well as $\mathrm{H}_{2} \mathrm{O}$ ice.

The mean aerosol particle size of the order of $0.30 \mu$ radius that is inferred by our results, could be considered also to be representative of a distribution of aerosol particle sizes between, say, 0.28 to $0.35 \mu$. It might be noted that this size range is quite unlike our terrestrial experience (Junge, 1967).

However, the total environment of Mars apparently is quite different from that of the Earth, and perhaps these results are not as unreasonable as they first may appear. Certainly, the limitations of the Mie scattering theory constraints must be recognized. In addition, our assumptions about a simple quiescent aerosol volume distribution are rightly questionable. Nevertheless, this study has improved the perspective concerning Mars, and hopefully enables one to discern what remains significant in the abundant speculation about the Martian atmosphere that pervades the literature.

\section{Acknowledgments}

We gratefully thank Mrs. Jaylee Mead (NASA-Goddard Space Flight Center) for her considerable assistance in performing and providing us with her Mie scattering computer calculations. The authors express their appreciation to the Grumman Aerospace Corporation for its sponsorship and encouragement in the pursuit of this study, and to their colleagues Dr. G. McCoyd, Dr. N. Milford, and L. Smith for stimulating discussions on various aspects of the problem.

\section{References}

Cann, M. W. P., Davies, W. O., Greenspan, J. A., and Owen, T. C.: 1965, NASA CR-298 Technical Report, IIT Research Institute.

Coulson, K. L.: 1969, Appl. Opt. 8, 1287.

de Vaucouleurs, G.: 1964, Icarus 3, 187.

Dollfus, A.: 1966, Compt. Rend. Acad. Soc. Paris 262, Serie b, 519. 
Dollfus, A. and Focas, J. H.: 1966, Polarimetric Study of the Planet Mars (AFCRL 66-492 (AD635, 928), Air Force Cambridge Research Laboratories, through European Office of Aerospace Research).

Egan, W. G.: 1967, J. Geophys. Res. 72, 3233.

Egan, W. G.: 1969, Icarus 10, 223.

Egan, W. G. and Becker, J. F.: 1969, Appl. Opt. 8, 720.

Egan, W. G. and Spagnolo, F. A.: 1969, Appl. Opt. 8 (in press).

Egan, W. G. and Foreman, K. M.: 1968, Astron. J. 78, S92.

Foreman, K. M.: 1969, AIAA Paper No. 69-52 (presented at the 7th Aerospace Sciences Meeting, New York City, Jan. 20-22, 1969).

Gierasch, P. and Goody, R.: 1967/1968, Planetary Space Sci. 15, 1465; 16, 615.

Johnson, F. S.: 1965, Science 150, 1445.

Junge, C.: 1967, NASA TTF-11, 091 (N67-36177).

Kaplan, L. D., Münch, G., and Spinrad, P.: 1964, Astrophys. J. 139, 1.

Kliore, A.: 1969, presentation of Mariners VI and VII radio occultation experiment results, NASA, Washington, D.C.

Kliore, A., Cain, D. L., and Levy, G. S.: 1966, Proc. 7th International COSPAR Symposium, Vienna, May 11-17, 1969.

Kuiper, G. P.: 1964, Comm. Lunar Planetary Lab. 2, 79.

Leighton, R. B., Horowitz, N. H., Murray, B. C., Sharp, R. P., Herriman, A. H., Young, A. T., Smith, B. A., Davies, M. E., and Leovy, C. B.: 1969, Science 166, 49.

Leovy, C. B. and Mintz, Y.: 1966, Memorandum RM-5110-NASA Contract No. NASr-21-(07), Rand Corporation, Santa Monica.

Mead, J. M. : 1970, this volume, p. 166.

O'Leary, B. T.: 1967, Space Science Laboratory Series 8, 103.

O'Leary, B. T. and Pollack, J. B.: 1969, Icarus 10, 238.

Rea, D. G. and O'Leary, B. T.: 1965, Nature 206, 1138. 